

\title{
Colloidal quantum dots for mid-IR applications
}

\author{
Emmanuel Lhuillier, Sean Keuleyan, Philippe Guyot-Sionnest
}

\section{To cite this version:}

Emmanuel Lhuillier, Sean Keuleyan, Philippe Guyot-Sionnest. Colloidal quantum dots for mid-IR applications. QSIP, 2012, Cargese, France. pp.133 - 136, 10.1016/j.infrared.2012.12.027 . hal-01438647

\section{HAL Id: hal-01438647 https://hal.science/hal-01438647}

Submitted on 25 Aug 2020

HAL is a multi-disciplinary open access archive for the deposit and dissemination of scientific research documents, whether they are published or not. The documents may come from teaching and research institutions in France or abroad, or from public or private research centers.
L'archive ouverte pluridisciplinaire HAL, est destinée au dépôt et à la diffusion de documents scientifiques de niveau recherche, publiés ou non, émanant des établissements d'enseignement et de recherche français ou étrangers, des laboratoires publics ou privés. 


\section{Colloidal Quantum Dots for Mid-IR Applications}

Emmanuel Lhuillier, Sean Keuleyan and Philippe Guyot-Sionnest

University of Chicago, James Franck institute, USA

929 E 57 th street, Chicago IL 60637, pgs@uchicago.edu

ABSTRACT : The use of colloidal material offers an interesting alternative to top down approaches for the realization of low cost infrared detectors. We demonstrate photoconduction in thin films of a colloidal material in the mid infrared (up to $7 \mu \mathrm{m}$ ), using $\mathrm{HgTe}$ colloidal quantum dots. Thin films of the colloidal quantum dots have a large absorption coefficient $\left(>10^{4} \mathrm{~cm}^{-1}\right)$, and the photoconductive response is dramatically improved by encapsulating the nanoparticle into an inorganic matrix of $\mathrm{As}_{2} \mathrm{~S}_{3}$. Such devices show fast response and large detectivity ( $>10^{10}$ jones) at temperatures above $200 \mathrm{~K}$.

Keywords: quantum dot, $\mathrm{HgTe}$, mid-infrared 


\section{INTEREST IN COLLOIDAL MATERIAL IN INFRARED DETECTION}

Infrared detection is currently driven by mature technologies, such as quantum well infrared photodetectors (QWIP) ${ }^{12}$, type II superlattices ${ }^{3}, \mathrm{HgCdTe}$ alloys or $\mathrm{InSb}^{4}$. In spite of up to fifty years of development, these materials remain expensive, limiting the use of the infrared cameras to primarily defense and astronomy applications. In addition, the push for higher operating temperature makes improvements even more challenging. In QWIP devices, the active layer is already operating close to its quantum limits $5,6,7,8$. New paradigms are thus necessary to achieve both higher operating temperature and reduced cost.

Quantum dot infrared detectors (QDIP) have offered an interesting alternative to quantum well device since the phonon bottle neck was expected to reduce the coupling between carriers and phonons, thus allowing higher operating temperature through a reduction of the dark current. In practice though, the epitaxial growth of the QD in its matrix keeps the coupling to the phonons high, and no significant dark current reduction has been achieved so far.

Colloidal quantum dots (CQD) show some evidence for a phonon bottle neck ${ }^{9}$, which may provide an advantage in devices. The absence of epitaxy between the dot and the surrounding matrix offers a better opportunity to reduce the coupling to phonons and indeed, slow relaxation has been observed in CQD, indicating that the discrete spectrum of the CQD is poorly coupled to the phonon bath. Moreover the synthesis and control of CQD material properties has seen tremendous development over the past two decades ${ }^{10}$. Control over properties in these materials is almost as advanced as that for molecular beam or metal organic chemical vapor deposition grown heterostructures. Nanoparticles can be zerodimentional (quantum dots ${ }^{11}$ ), one dimentional (nanorods ${ }^{12}$ ) or two dimentional (nanoplatelets ${ }^{1314}$ ). Core-shell heterostructures are also synthetized with type $\mathrm{I}^{15}$ and type $\mathrm{II}^{16}$ band alignments. The use of semiconductor nanocrystals for optoelectronic applications ${ }^{17}$ has been demonstrated in solar cells ${ }^{18}$, LEDs and detectors ${ }^{19}$. Materials and such devices though have been developed primarily for visible applications with less work devoted to the infrared (IR).

A possible path to access the mid-IR range of wavelengths is based on intraband transitions in wide band gap materials such as $\mathrm{CdSe}^{20}$. To realize these transitions, the conduction band can be populated with electrons (or the valence band with holes) by gating or optical pumping. This requirement, though, increases complexity and may be incompatible with many applications. Near-IR energiesare accessed with lead chalcogenide CQD ( $\mathrm{PbS}, \mathrm{PbSe}, \mathrm{PbTe})$. Their use in converting the near-IR part of the solar spectrum and in infrared detection has been reported by Konstantatos et a/1921. The long wavelength limitwith these lead based CQD remains restricted by their bulk band gap. ${ }^{22}$ Semimetals, such as HgTe are consequently the best candidates for CQD devices for the mid wavelength infrared (MWIR). Many works have reported the synthesis of $\mathrm{HgTe}$ for near-IR applications ${ }^{232425}$, but none of them have reached cut-off wavelengths up to the mid-IR, mostly due to difficulties in preparing stable, large HgTe CQD. In addition, the use of CQD in MWIR remains very challenging due to the capping organic ligands, which have vibrational modes in this range of energy.

In this paper we discuss the synthesis and photoconduction properties of CQD material in the MWIR. In Section 2 we discuss the chemical synthesis of the HgTe CQD as well as the device preparation and experimental setup. In the third section we focus our interest on the optical properties (relation size-absorption edge wavelength and absorption coefficient). The final section deals with the photoconduction properties of thin films of $\mathrm{HgTe}$ nanoparticles. First, we underline the possible difficulties of dealing with colloidal material in the mid-IR, then we show the limits of a photoconductive response with an organic ligand matrix. Finally we demonstrate that embedding the HgTe CQD into a matrix of $\mathrm{As}_{2} \mathrm{~S}_{3}$ drastically improves carrier mobility and consequently the film responsivity and detectivity. 


\section{MATERIAL AND DEVICES}

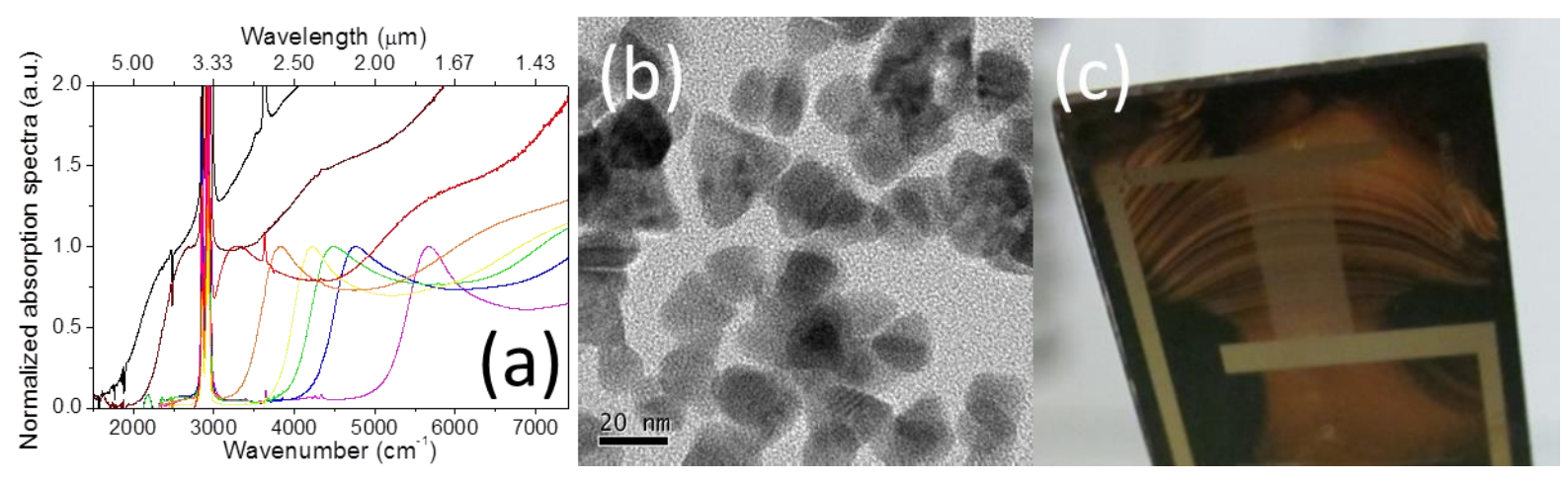

Figure 1(a) Absorption spectrum for HgTe CQD solution in the IR range for several particle sizes between 5 and $15 \mathrm{~nm}$. The sharp feature near $2900 \mathrm{~cm}-1$ is from the capping ligands. (b) TEM image of $\mathrm{HgTe}$ nanoparticles. (c) Photo of a film of HgTe deposited over interdigitated electrodes.

The material synthesis is performed following the recipe developed in references

26,27. A first generation of material26 was developed and showed tunable optical properties in the MWIR. The particles were found to be aggregated and the optical spectra were quite broad. In a later development, a new synthetic method ${ }^{27}$ was developed to improve the CQD monodispersity and as a consequence the sharpness of the optical spectra. Briefly, $\mathrm{HgCl}_{2}$ is dissolved in octadecylamine, the mixture is heated to $100^{\circ} \mathrm{C}$ for one hour under vacuum. Then a solution of $\mathrm{Te}$ in trioctylphosphine (TOP) (TOPTe) is quickly added to the flask. The solution darkens, indicating the formation of HgTe. The particle size is controlled by the duration and temperature of the reaction, where increasing the duration or the temperature leads to an increase of the size. The obtained solution is quenched into a room temperature solution of TOP, dodecanethiol and tetrachloroethylene (TCE). The absorption spectra shown in Figure 1 (a) shows the possibility to tune the exciton feature wavelength at room temperature between 1.5 and $5 \mu \mathrm{m}$.

Typical particles are shown in a transmission electron microscopy (TEM) image in Figure 1 (b). CQD are typically composed of an inorganic core (i.e. the semiconductor $\mathrm{HgTe}$ in this case) surrounded by an organic shell of ligands, which control the growth and ensure colloidal stability of the solution. As will be discussed later, the presence of this organic shell makes the use of this material in the IR challenging.

Films of CQD can be obtained by dropcasting, spincoating or dipcoating. In order to obtain homogeneous film, the CQD initially dissolved into TCE are precipitated by addition of an alcohol and centrifuged. The obtained solid is redissolved into a 9:1 in volume mixture of hexane:octane. This film can be dropcast over interdigitated electrodes (50 pairs of $5 \mathrm{~mm}$ long Pt electrodes with a $10 \mu \mathrm{m}$ spacing), see Figure 1 (c). For a single deposition the film thickness is typically $100 \mathrm{~nm}$. Multilayer deposition is possible by fixing each layer.This can be achieved by a ligand exchange process where the relatively long ligands (eg. dodecanethiol) are replaced by shorter ones such as ethanedithiol. For electrical characterization the sample is mounted on the coldfinger of a closed cycle He cryostat.

For characterization the I-V curves are acquired using a Keithley 6487 picoammeter. Photocurrent spectra are obtained by biasing the sample using a femto DLCPA 200 amplifier, and the amplified output is processed via a Nicolet Magna IR 550 FTIR. The time response is measured by illuminating the device with a $10 \mathrm{ps}$ pulse from a $1.06 \mu \mathrm{m}$ Nd:YAG laser, with the sample biased by a battery, and the response recorded through a Tektronik TDS 1012B oscilloscope. The responsivity of the system is 
measured while the system is illuminated by a blackbody at $955^{\circ} \mathrm{C}$ and the incoming light is mechanically chopped at $25 \mathrm{~Hz}$. The optical aperture of the system is $f / 3$. The biasing, magnification of the signal and the acquisition are made with the amplifier and oscilloscope previously mentioned. Noise is measured in the dark for the evaluation of the detectivity. The noise measurements are obtained by biasing and amplifying the signal using the same amplifier as above and the data is acquired with a Stanford SR 760 spectrum analyzer.

\section{OPTICAL PROPERTIES}

As for quantum well or superlattice based devices the optical properties are tuned through a change of the size of the system. The confinement takes the same role as the cadmium composition in the $\mathrm{HgCdTe}$ alloy to tune the band gap energy of the material. Tuning the size of the particle from 5 to 15 $\mathrm{nm}$ results in the cut-off wavelength tuning from 1.5 to $5 \mu \mathrm{m}$ at room temperature, as shown in Figure 2 (a). This is in good agreement with a prediction coming from a two band $k \cdot p$ model ${ }^{28}$. Pushing the absorption out to the long wavelength infrared (LWIR) will require the growth of CQD as large as $18 \mathrm{~nm}$.

An alternative way to the tune the band edge energy is to change the temperature of the sample. As for bulk HgTe, The HgTe CQD have a positive value of $\frac{d E_{B E}^{Q D}}{d T}$, meaning that a cooling of the material leads to a redshift of the transition. As an example, sample with a cutoff wavelength of $5 \mu \mathrm{m}$ at room temperature absorbs up to $7 \mu \mathrm{m}$ at liquid nitrogen temperature. This shift of the band gap energy with temperature exhibits a size dependence which is attributed to the tuning of the relative weight of the inter- and intraband coupling of the carriers to the optical phonons.

For application in photodetectors, a key parameter is the absorption coefficient. In our case the absorption coefficients are measured by depositing a film of CQD onto a Si wafer and measuring the film thickness by ellipsometry (Gartner L116S), while the absorption is obtained through a FTIR spectrometer (Nicolet Magna IR 550). The absorption coefficients measured are typically in the $10^{4}$ $\mathrm{cm}^{-1}$ range (see Figure 2 (b)), which is as high as for the bulk $\mathrm{HgTe}^{29}$ and $\mathrm{HgCdTe}$ alloy ${ }^{303132}$. Compared to QDIP obtained through Stransky-Krastanov growth, these values are considerably higher, due to the larger QD packing density in the colloidal thin films. 

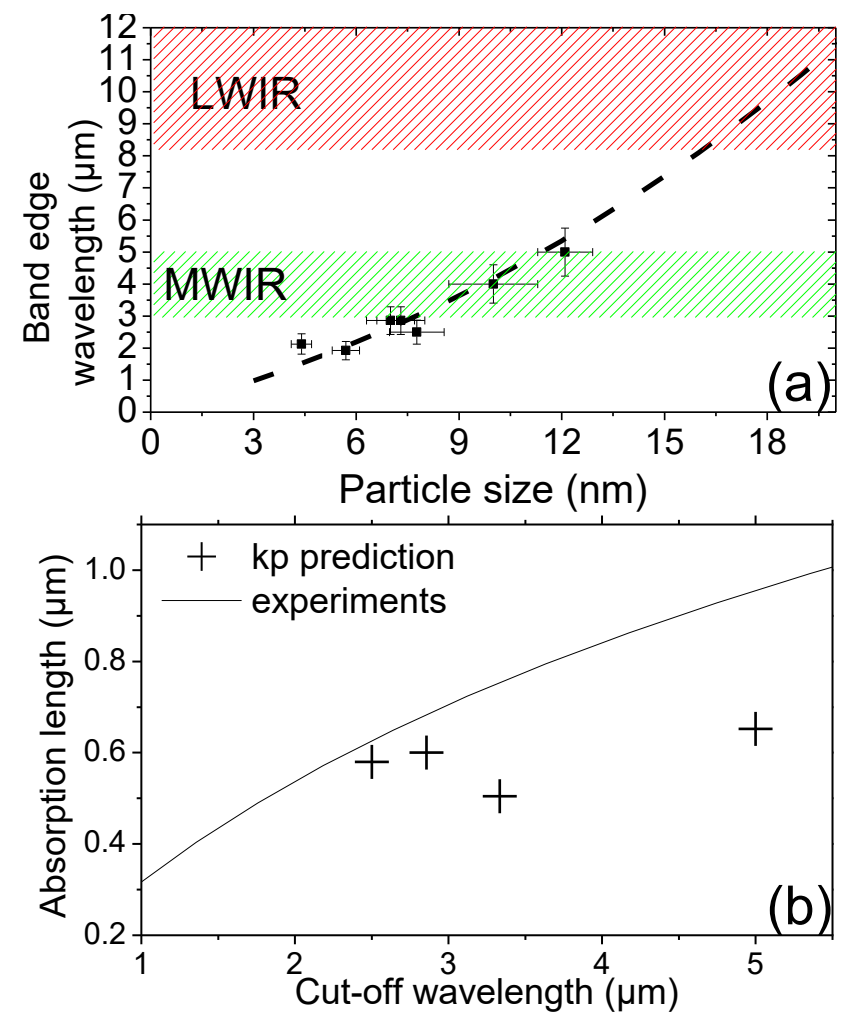

Figure 2 (a) $\mathrm{k} \cdot \mathrm{p}$ modeling of cut-off wavelength of $\mathrm{HgTe} C Q D$ as a function of their size. Experimental data appears as cross. (b) Absorption length as a function of the cut-off wavelength. The continuous line is obtained from $\mathrm{k} \cdot \mathrm{p}$ modeling.

\section{PHOTOCONDUCTION AND DEVICES PERFORMANCE 4.1. Challenges for CQD in mid-infrared}

Absorption in the mid-IR has been reported recently with other colloidal material ${ }^{33}$, nevertheless obtaining photoconduction remain even more challenging. Here, we first discuss some of the difficulties in realizing IR photoconduction in films of CQD.

- CQD are composed of an inorganic semiconductor core surrounded by organic ligands, which control the growth and the colloidal stability of the nanoparticle. These ligands are typically long alkane chains which are connected to the core through an organic functional group such as an acid, amine, or thiol. The ligands have vibrational transitions in the mid-IR, (eg. the C-H bond has a resonance near $3000 \mathrm{~cm}^{-1}$ ). Energy can therefore be lost from the electronic excitation in the core, to the organic ligand vibrations. Such a process is, for example, known to quench the photoluminescence of CQD in this range of wavelength ${ }^{34}$. Removing/replacing these organic molecules is therefore desired.

- Typical organic ligands are also very poorly conductive, since they behave as tunnel barrier for the inter CQD transport. Carrier mobilities in drop-cast films of CQD are typically around 10${ }^{6} \mathrm{~cm}^{2} \mathrm{~V}^{-1} \mathrm{~s}^{-1}$, limiting the photoconductive responsivity.

- Depending on the strength of the binding to the semiconductor core, the ligands can be removed which result in incomplete passivation of the particle surface, resulting in the formation of trap states which can further alter mobilities and recombination rates.

- Finally a CQD film can be seen as a bulk material full of cracks, which can lead to additive electrical $1 / \mathrm{f}$ noise ${ }^{35}$ and limited detectivity.

To achieve detectors with large detectivity in the mid-IR all these points have to be addressed. 


\subsection{Limitations of the organic capping}

The first strategy to address the previously mentioned issues is the now commonly applied ligand exchange, which aims to reduce the length of the ligands.. Typically, the initial ligands with twelve or more carbons are chemically replaced by shorter ligands (with four or less carbons). This process is experimentally very easy to perform - simply dipping the CQD film into a solution of short ligands in ethanol efficiently achieves the exchange. The number of absorbing $\mathrm{C}-\mathrm{H}$ bond is reduced and the mobility drastically increased due to the reduced interparticle distance. Ligand exchange with ethanedithio ${ }^{36}$ has been extensively investigated and typically increases the conductance by 3 orders of magnitude.

The ligand exchange process, performed on our films of $\mathrm{HgTe} C Q D$ gave an increase in mobility, accompanied though, by a non-monotonic temperature dependence of the dark current (see Erreur ! Source du renvoi introuvable. (a)). In addition, responsivities remained low $\left(\approx 1 \mathrm{~mA}^{-1} \mathrm{~W}^{-1}\right.$, see Erreur ! Source du renvoi introuvable. (b)), a consequence of the mobility increasing to only about $10^{-4} \mathrm{~cm}^{2} \mathrm{~V}^{-1}$ $\mathrm{s}^{-1}$.
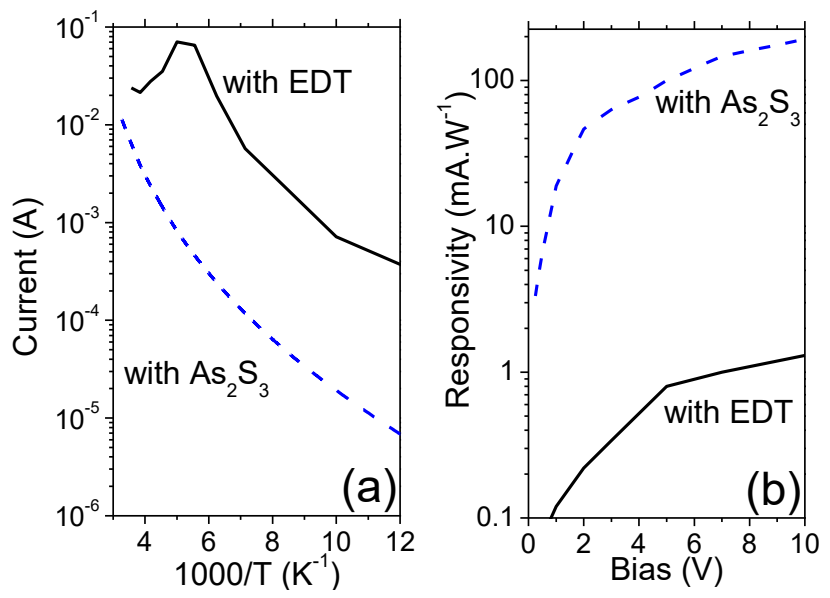

Figure 3 (a) Current as a function of the inverse of the temperature for a film of $\mathrm{HgTe}$ CQD processed in air with EDT and processed air free with $\mathrm{As}_{2} \mathrm{~S}_{3}$ under $1 \mathrm{~V}$ bias. (b) The responsivity for the same two films at room temperature.

\subsection{Encapsulation into an inorganic matrix}

To obtain further improvement of the responsivity and detectivity, we wish to completely remove the organic component of the material, which is poorly conductive and leads to poor passivation. We identify $\mathrm{As}^{3+}$ as an ion which largely increases the conductance of the $\mathrm{HgTe} C Q D$ film and $\mathrm{As}_{2} \mathrm{~S}_{3}$ as a possible IR transparent matrix ${ }^{37}$ including this ion with good optical properties in the mid-IR. Once the material is processed air-free (to avoid the activation of acceptor states) and encapsulated into by $\mathrm{As}_{2} \mathrm{~S}_{3}{ }^{38}$, we recover an intrinsic behavior for the temperature dependence of the current, see Erreur ! Source du renvoi introuvable. (a). Moreover, a responsivity one hundred times large is obtained, see Erreur! Source du renvoi introuvable. (b), and can be attributed to the increase of the carrier mobility. In addition, we note that the frequency cut-off remains high $(>100 \mathrm{kHz}$, see Figure 4 (a)) and that the measured detectvity can be as high as $10^{10}$ Jones, (Figure 4 (b)). 

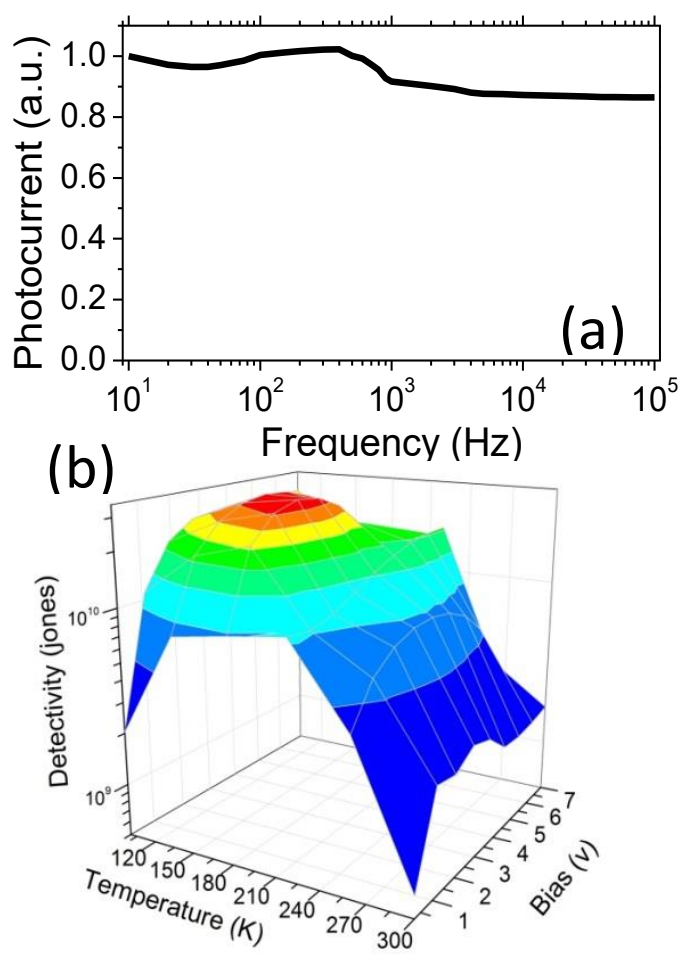

Figure 4 (a) Photocurrent as a function of the modulation frequency of a $800 \mathrm{~nm}$ laser for a film of $\mathrm{HgTe} C Q D$ processed air free with $\mathrm{As}_{2} \mathrm{~S}_{3}$. (b) Measured detectivity, as a function of temperature and bias, of a film of $\mathrm{HgTe} C Q D\left(\lambda_{\text {cut-off }}=3 \mu \mathrm{m}\right)$ processed air-free and with $\mathrm{As}_{2} \mathrm{~S}_{3}$.

\section{CONCLUSION}

We report the synthesis, optical, and electro-optical characterization of mid-infrared active HgTe CQD with a band gap tunable through the near- and mid-infrared. This material can be prepared easily via standard solution synthesis. Thin films have a large absorption coefficient $\left(>10^{4} \mathrm{~cm}^{-1}\right)$, and once processed air free and encapsulated in the right inorganic matrix this material presents a large detectivity ( $>10^{10}$ jones) and fast time response. This progress paves the way for the use of this new material in low cost infrared detectors. 


\title{
6. REFERENCES
}

\author{
${ }^{1}$ H. Schneider and H.C. Liu, in Quantum well infrared photodetectors - Physics and applications \\ (Springer, Heidelberg, 2006). \\ 2 B.F. Levine, J. Appl. Phys. 74, R1 (1993). \\ ${ }^{3}$ D. H. Chow, R. H. Miles, J. N. Schulman, D. A. Collins and T. C. McGill, Semicon Sci. Technol. 6, \\ C47 (1991). \\ ${ }^{4}$ A. Rogalski, Infrared Phys. Techn. 43, 187 (2002). \\ ${ }^{5}$ E. Lhuillier, I. Ribet-Mohamed, A. Nedelcu, V. Berger and E. Rosencher, Phys. Rev. B 81, 155305 \\ (2010)
}

6 E. Lhuillier, I. Ribet-Mohamed, N. Péré-Laperne, M. Tauvy, J. Deschamps, A. Nedelcu, E. Rosencher, Infrared phys. tech. 53, 425 (2010).

7 E. Lhuillier, I. Ribet-Mohamed, M. Tauvy, A. Nedelcu, V. Berger and E. Rosencher, Infrared phys. tech. 52, 132-137 (2009).

8 E. Lhuillier, I. Ribet-Mohamed, E. Rosencher, G. Patriarche, A. Buffaz, V. Berger, and M. Carras, App. Phys. Lett. 96, 061111 (2010).

9 A. Pandey and P. Guyot-Sionnest, Science 322, 929 (2008).

10 V. Klimov, Nanocrystal Quantum Dots, Second Edition, CRC press, Boca raton (2010).

11 C.B. Murray, D.J. Norris, M.G. Bawendi, J Am Chem Soc 115, 8706 (1993).

12 L. Manna, E. Scher, A.P. Alivisatos, J AM. Chem. Soc. 122, 12700 (2000).

${ }^{13}$ S. Ithurria, M. D. Tessier, B. Mahler, R. P. S. M. Lobo, B. Dubertret and Al. L. Efros, Nature

Materials 10, 936 (2011).

${ }^{14} \mathrm{~S}$. Ithurria, G. Bousquet and B. Dubertret, J Am Chem Soc. 133, 3070 (2011).

${ }^{15}$ M. A. Hines and P. Guyot-Sionnest. J. Phys. Chem. 100, 468 (1996)

${ }^{16}$ S. Kim, B. Fisher, H.J. Eisler and M. Bawendi, J. Am. Chem. Soc. 125, 11466 (2003).

17 D.V. Talapin, J.S. Lee, M. V. Kovalenko, and E.V. Shechenko, Chem. Rev.110, 389 (2010)

${ }^{18}$ A. J. Nozik, Quantum dot solar cells, Physica E 14, 115 (2002).

19 G. Konstantatos, I. Howard, A. Fischer, S. Hoogland, J. Clifford, E. Klem, L. Levina, and E.H.

Sargent, Nature 442, 180 (2006).

20 P. Guyot-Sionnest and M. A. Hines, Appl. Phys. Lett. 72, 686 (1998).

21 G. Konstantatos, E. H. Sargent Infrared Physics and Technology, vol. 54, 278 (2011).

22 J.M. Pietryga, R.D. Schaller, D. Werder, M.H. Stewart, V.I. Klimov and J.A. Hollingsworth, J. Am.

Chem. Soc. 126, 11752 (2004).

${ }^{23}$ M.V. Kovalenko,E. Kaufmann, D. Pachinger, J. Roither, M. Huber, J. Stangl, G., Hesser, F. Schäffler and W. Heiss, J. Am. Chem. Soc. 128, 3516 (2006).

${ }^{24}$ M.T. Harrison, SV Kershaw, MG Burt, A Rogach A. Eychmüller and H. Weller, J. Mater. Chem. 9, 2721 (1999).

${ }^{25}$ A. Rogach, S. Kershaw, M. Burt, M. Harrison, A. Kornowski, A. Eychmuller and H. Weller, Adv. Mater. 11, 552 (1999).

${ }^{26}$ S. Keuleyan, E. Lhuillier, V. Brajuskovic and P. Guyot-Sionnest, Nat Photon 5, 489 (2011).

27 S. Keuleyan, E. Lhuillier and P. Guyot-Sionnest, J. Am. Chem. Soc 133, 16422 (2011).

${ }^{28}$ E. Lhuillier, S. Keuleyan and P. Guyot-Sionnest, nanotechnology 23, 175705(2012).

29 P. Man, and D.S. Pan, Phys. Rev. B 44, 8745 (1991).

30 M.D. Blue, Phys. Rev. 134, A226 (1964).

${ }^{31}$ P. Norton, Opto-Electronics Rev. 10, 159 (2002).

32 A Rogalski, Rep. Prog. Phys. 68, 2267(2005).

${ }^{33}$ A.Sahu, A. Khare, D. D. Deng and D. J. Norris, Chem. Commun. 48, 5458 (2012).

${ }^{34}$ H. Liu and P. Guyot-Sionnest, J. Phys. Chem. 114, 14860 (2010).

${ }^{35}$ E. Lhuillier, S. Keuleyan, P. Rekemeyer and P. Guyot-Sionnest, J. Appl. Phys 110, 032110 (2011).

${ }^{36}$ M. Law, J.M. Luther, Q. Song, M.C. Beard and A. Nozik, ACS Nano 2, 271 (2008).

${ }^{37}$ M. V. Kovalenko, R. D. Schaller, D. Jarzab, M. A. Loi and D. V. Talapin, J. Am. Chem. Soc. 134, 2457 (2012).

${ }^{38}$ E. Lhuillier, S. Keuleyan, P. Zolotavin and P. Guyot-Sionnest, accepted in adv. Mat. (2012). 